\title{
La cirugía como una sinfonía. Un proyecto para el trabajo en equipo y coordinado
}

\author{
Surgery as a symphony. A project for team and coordinated work
}

\author{
Adonis T. Ramírez $\mathbb{D}$ \\ Médico, especialista en Cirugía General y Cirugía de Cabeza y Cuello, Clínica Medilaser, Neiva, Colombia.
}

\section{Resumen}

La seguridad del paciente es uno de los aspectos de mayor relevancia en la atención en un quirófano. El trabajo en equipo y coordinado, sumado al liderazgo, permite que los errores sean menos. Mantener un equipo estable en el quirófano, que conozca bien los procedimientos, y donde cada participante sabe el rol que juega, es uno de los factores más importantes para lograr un trabajo eficiente, con disminución de las complicaciones y del tiempo quirúrgico. Comparar el trabajo en el quirófano con una orquesta sinfónica, nos ayuda a entender la importancia del trabajo coordinado.

Palabras Clave: cirugía general; quirófanos; equipo quirúrgico; liderazgo; ambiente de trabajo.

\begin{abstract}
Patient safety is one of the most important aspects of care in an operating room. Coordinated teamwork, coupled with leadership, allows mistakes to be fewer. Maintaining a stable team in the operating room, who knows the procedures well, and where each participant knows the role they play, is one of the most important factors to achieve efficient work, with a reduction in complications and surgical time. Comparing work in the operating room with a symphony orchestra helps us understand the importance of coordinated work.
\end{abstract}

Keywords: general surgery; operating rooms; surgical equipment; leadership; working environment.

Fecha de recibido: 28/05/2020 - Fecha de aceptación: 12/06/2020

Correspondencia: Adonis T. Ramirez, Calle 5 \# 28-05 apartamento 402, Neiva, Colombia. Teléfono: 320-3393506.

Correo electrónico: adonistupac@gmail.com

Citar como: Ramírez AT. La cirugía como una sinfonía. Un proyecto para el trabajo en equipo y coordinado.

Rev Colomb Cir. 2020;35:550-2. https://doi.org/10.30944/20117582.673

Este es un artículo de acceso abierto bajo una Licencia Creative Commons - BY-NC-ND https://creativecommons.org/licenses/by-ncnd/4.0/deed.es 
La seguridad del paciente se ha convertido en un componente esencial en la asistencia sanitaria. El trabajo realizado en un quirófano es un trabajo complejo, que no solo requiere la capacitación científica de los profesionales sino, además, la consecución de otras habilidades, como trabajo en equipo, comunicación, respuesta adecuada ante situaciones de estrés y capacidad de liderazgo ${ }^{\mathrm{I}}$. Aproximadamente la mitad de los errores hospitalarios ocurren en el quirófano o en las salas de cuidado postoperatorio, y la mayoría se deben a problemas de comunicación y falta de liderazgo ${ }^{2-4}$.

La sinfonía es música para orquesta, incluyendo de cuerda o de cámara, que constituye una unidad estructural, entendiéndose como un todo, o sea, que no pueden separarse sus partes. La sinfonía clásica consta de tres o cuatro movimientos. La más completa es la que consta de 4 movimientos, que son los siguientes:

- Un movimiento inicial rápido, llamado allegro, sobre el cual se construirá el resto. Para dar un carácter unitario a la obra, algunas veces sus temas son usados en otros movimientos, especialmente en el último. Su tonalidad principal se indica en el título de la obra.

- El segundo movimiento es lento, adagio, con tonalidad opuesta, menor o mayor, a la del primer movimiento; su forma es menos definida.

- El tercer movimiento es un minuetto, o scherzo desde la época de Beethoven. El scherzo tiene un carácter alegre y rítmico, una especie de broma musical. Su tonalidad puede escogerse libremente, y su forma no es tan rigurosa como el minuetto, ni tiene que adoptar su ritmo, pero suele tener estructura ternaria, con la parte central también llamada trío.

- El cuarto movimiento, finale, es rápido, normalmente un allegro o presto, escrito en la tonalidad principal.

La orquesta sinfónica o filarmónica es una agrupación o conjunto musical de gran tamaño, generalmente con más de ochenta músicos en su lista, que cuenta con varias familias de instrumentos musicales, como viento madera, viento metal, percusión y cuerda. Sólo en algunos casos llega a tener más de cien, pero el número de músicos empleados en una interpretación particular puede variar según la obra que va a ser ejecutada. Cada integrante interpreta un solo instrumento, para el cual es un experto, pero participa en diferentes piezas.

El director cumple con una función principal respecto a la orquesta, puesto que es el que la dirige e incluso da la formación musical. Es una persona que no sólo mantiene el tiempo de la pieza y da las entradas de los instrumentos para que la interpretación sea coherente, sino que debe interpretar la partitura según el concepto "global", manteniéndose fiel al espíritu original de la obra, pero dando una visión personal. Para conseguirlo, debe conocer en profundidad la vida y obra de los compositores.

El acto quirúrgico es similar a una sinfonía; es una actividad que implica, desde mi propuesta, cuatro tiempos o movimientos equiparables, que son los siguientes:

- Valoración preoperatoria, con una consulta especializada, para diagnóstico y orientación de la terapia quirúrgica, donde se explican el procedimiento quirúrgico y sus posibles complicaciones, los tratamientos alternativos y los riesgos del procedimiento, y se diligencia y firma el consentimiento informado. Incluye la consulta anestésica preoperatoria, para valorar el riesgo anestésico, explicar el tipo de la anestesia y sus complicaciones, y firmar el consentimiento.

- Ingreso a salas de cirugía: en este momento se diligencia la historia clínica de ingreso, se verifica la documentación, las autorizaciones, los resultados de exámenes diagnósticos y la valoración preoperatoria.

- Tiempo operatorio: se realiza la lista de chequeo de seguridad del paciente, con la pausa preoperatoria, donde se verifica la identificación del paciente, la cirugía que se va a 
realizar y la lateralidad, además de que se cuente con las ayudas diagnósticas y el material quirúrgico para el procedimiento, y se discuten las posibles complicaciones.

- Recuperación posanestésica: valoración en sala de recuperación para evitar complicaciones ventilatorias y diagnosticar de manera oportuna alguna complicación, como hemorragia postoperatoria. Finalmente se explica al paciente y sus familiares el procedimiento y los resultados.

Los integrantes del equipo quirúrgico (auxiliar de enfermería, instrumentador, ayudante quirúrgico, anestesiólogo y cirujano) deben ser expertos en su materia y conocer perfectamente los procedimientos quirúrgicos que se realizan, así como las condiciones y necesidades de cada uno (posición del paciente, requerimientos de dispositivos especiales, instrumental necesario, entre otros), asemejándose a los músicos de una orquesta sinfónica. En el quirófano también se cuenta con un director, que puede ser el cirujano o el anestesiólogo, un rol que se comparte dentro de la sala.

El trabajo en equipo implica unos objetivos comunes y unas funciones específicas de cada miembro, esto requiere capacidad de liderazgo, buena comunicación y una adecuada coordinación. Cuando los integrantes de un equipo de trabajo en el quirófano se mantienen, esto redunda en el conocimiento detallado del proceso, experticia y adecuada comunicación, demostrando en diferentes estudios que la eficiencia operatoria aumenta, mientras los errores y las complicaciones disminuyen ${ }^{5}$. Si se alcanza un comportamiento en salas de cirugía como una orquesta sinfónica, se lograrían las siguientes ventajas:

I. Disminución de complicaciones

2. Disminución del tiempo intraoperatorio

3. Manejo ambulatorio de los pacientes.

4. Equipos unificados.
5. Guías de manejo y de procedimientos estandarizados

6. Disminución de costos

7. Uso racional de los recursos

Quedan los interrogantes de la aplicabilidad de un sistema como este en las diferentes instituciones hospitalarias, donde a veces se tiene una alta rotación del personal, lo que implica que no haya una "especialidad" para realizar un trabajo en equipo coordinado, eficiente y cordial.

\section{Consideraciones éticas}

Consentimiento informado: Este artículo parte de una revisión de la literatura, y como tal no hay necesidad de un consentimiento informado ni de aprobación del Comité de Ética Institucional.

Conflicto de interés: El autor declara no poseer ningún conflicto de intereses.

Fuente de financiación: Recursos propios del autor.

\section{Referencias}

I. Cassinello-Plaza F. La importancia del trabajo en equipo en las salas de cirugía. Rev Colomb Anestesiol. 20I5;43:I-2. http://dx.doi.org/Io.IOI6/j.rca.20I4.IO.003

2. Leape LL, Brennan TA, Laird N, Lawthers AG, Localio $\mathrm{AR}$, Barnes BA, et al. The nature of adverse events in hospitalized patients. Results of the Harvard Medical Practice Study II. N Engl J Med. 199I;324:377-84. http://doi.org/IO.IO56/NEJMi99IO2073240605

3. Pronovost PJ, Thompson DA, Holzmueller CG, Lubomski LH, Dorman T, Dickman F, et al. Toward learning from patient safety reporting systems. J Crit Care. 2006;2I:305-I5.

http://doi.org/IO.IOI6/j.jcrc.2006.07.00I

4. Bejarano M. Los cirujanos y el trabajo en equipo. Rev Colomb Cir. 2019;34:II2-3. https://doi.org/I0.30944/20117582.IO4

5. Maruthappu M, Duclos A, Zhou CD, Lipsitz SR, Wrigth J, Orgill D, Carty MJ. The impact of team familiarity and surgical experience on operative efficiency: a retrospective analysis. J R Soc Med. 20I6;I09:I47-53. https://doi.org/Io.II77/oI4IO768I66343I7 